\title{
LIQUIROB - A NEW SOLUTION FOR ADVANCED SHROUD MANIPULATION - FROM AN IDEA TO AN INDUSTRIAL SOLUTION IN 3 MAJOR STEPS*
}

\author{
Juergen Meisel ${ }^{1}$ \\ Wilhelm Altreiter ${ }^{2}$ \\ Simon Pfeil \\ Gerald Prin ${ }^{4}$ \\ Roger Scheidegger ${ }^{5}$ \\ Oliver Lang ${ }^{6}$
}

\begin{abstract}
The paper will explain how to successfully implement a robotic solution from the initial idea to a working industrial solution. Primetals Technologies can call upon 10 years of experience in installation of Robot solutions in the harsh environment of steelmaking plants - the so-called LiquiRob. To enhance an existing shroud manipulation system, the idea to connect the shroud with a bayonet mechanism to the ladle nozzle was born in accordance with the requirements of a well-known steel producer. The paper will describe the development steps from the first simulations and prototype testing in the laboratory, it will show the intensive system testing under workshop conditions and will finally depict the successful integration into the plant. Furthermore, operational experience will be shown as well as the advantages of such an installation compared to other shroud manipulation systems.
\end{abstract}

Keywords: Continuous casting, shroud, robot.

1 Head of Mechatronic Products for CCM, Primetals Technologies Austria GmbH, Turmstrasse 44, 4031 Linz, Austria.

2 Ing, Project Manager, Primetals Technologies Austria GmbH, Turmstrasse 44, 4031 Linz, Austria.

3 DI,Robotics and Machine Vision, Primetals Technologies Austria GmbH, Turmstrasse 44, 4031 Linz, Austria.

4 Ing, Automation Engineer, Primetals Technologies Austria GmbH, Turmstrasse 44, 4031 Linz, Austria.

5 Mechanical Design Engineer, Primetals Technologies Austria GmbH, Turmstrasse 44, 4031 Linz, Austria.

6 DI Dr.,Innovation Manager, Primetals Technologies Austria GmbH, Turmstrasse 44, 4031 Linz, Austria. 


\section{INTRODUCTION}

The ladle operation platform in general is not a very good working place for humans. There is a lot of heat and dust, steel is splashing and people can get hurt by parts falling from the ladle rim.[2]

Customers are aware of this danger and furthermore they want to ensure that an emergency turning of the ladle turret is possible at any time. Actually used shroud manipulation systems can be a risk concerning this topic. Therefore they were looking for a new shroud manipulator that cannot block the ladle.

At the same time, Primetals finished the basic development of the new bayonet shroud holding system. After presenting the prototype in our workshop in Linz, a contract for ladle operation platform LiquiRob for the customer was signed.

\section{DESIGN}

\subsection{Bayonet}

The main component is the new bayonet that guarantees a tight connection of the shroud to the ladle nozzle without any manipulator [4]. It consists of two main parts: The shroud holder ladle part is mounted on each ladle. It typically has 3 pins that are used as mechanical connection to the moveable shroud holder (Figure 1).

The shroud is placed into the shroud holder. LiquiRob brings it to the ladle, and shroud holder and shroud holder ladle part are connected like a lens to an SLR camera. While the connection between shroud holder and shroud holder ladle part is mechanically defined, the force with which the shroud is pressed against the ladle nozzle can be adjusted by the springs (typically 4-10 springs) on the shroud holder that apply that force. With a higher number of springs, there is the additional advantage of redundancy of springs.

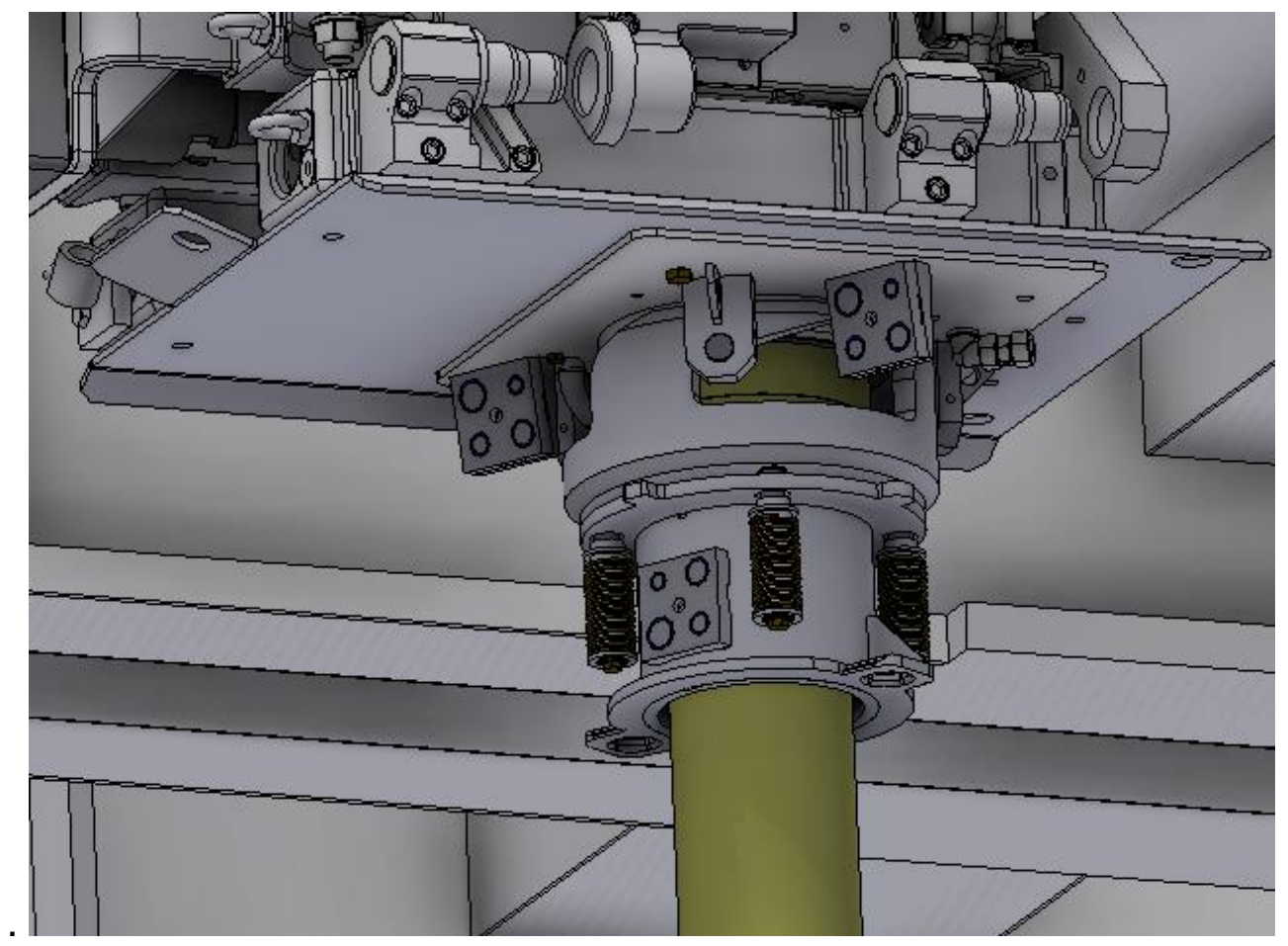

Figure 1. Basic design of shroud holder and shroud holder ladle part. 
Holding and manipulation of the complete system is done by the Shroud gripper which is the according tool on LiquiRob. With the length of the shroud gripper, a high

transmission ratio can be achieved, so a small robot force can generate high torques on the shroud holder.

The main advantages of this concept are:

- The manipulator that brings the shroud to the ladle, in this case PT LiquiRob, is not connected to the ladle

- So LiquiRob can perform other tasks, too.

- The force with which the shrouds are pressed against the ladle is always constant, but can be adjusted by the design of springs

- No movement between shroud and holder when slide gate is moving.

- There is no need to have special shrouds, like for other automatic shroud holding systems. The holder is designed for customers' shroud types. So the customers still has flexibility in choosing different shroud suppliers.

- Even the slide gate can remain the same.

- The mechanics that have to be mounted on the ladle respectively the slide gate are simple and cheap

- The complete system guarantees a quick and reliable shroud exchange

\subsection{SOS Shroud}

The SOS shroud (Figure 2) is an innovative method for opening frozen ladles.

Basics are the conventional shroud and PT shroud holder. Then a telescope tube gets inserted into the shroud and connected with the oxygen tube. This then has a connector through which LiquiRob is supplying oxygen. All assembly of SOS shroud is done in the maintenance area prior to use.

So if opening of ladle does not work, LiquiRob connects the SOS shroud to the ladle and supplies oxygen to the SOS shroud holder. By supplying oxygen the telescope tube is moved upwards (against the ladle) and the oxygen flame is ignited.

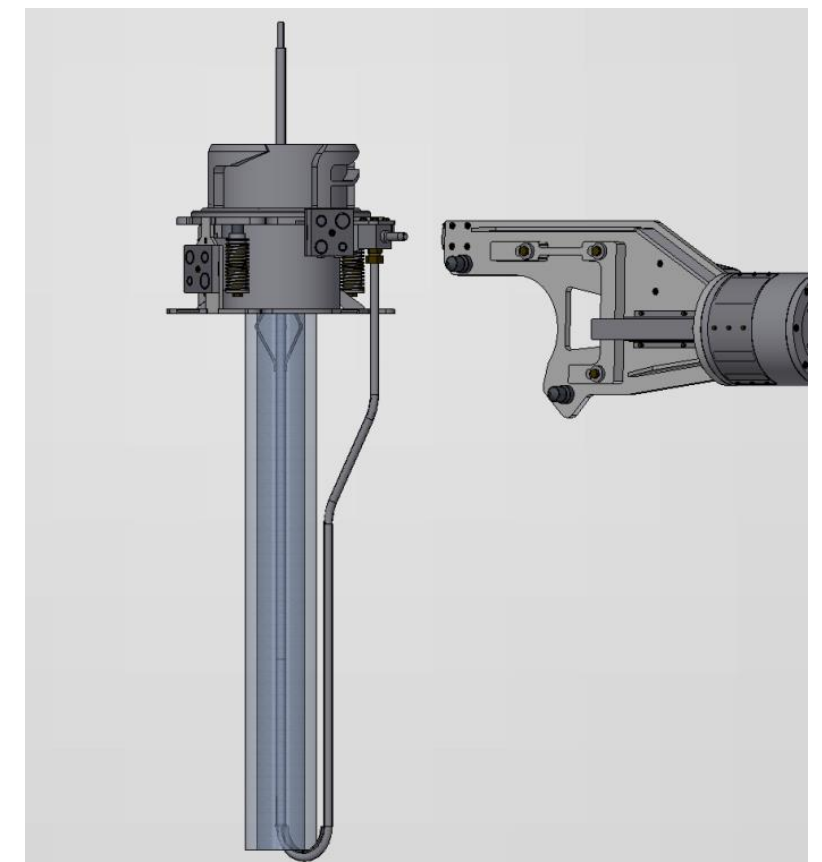

Figure 2. SOS shroud with coupling system for oxygen supply by LiquiRob 
The SOS shroud holder has an additional connection for oxygen supply

The big advantages of this system are:

- Less oxygen in steel because shroud is already attached when ladle opens

- Closing of slide gate to attach the shroud is not necessary and therefore risk for freezing again is eliminated

- Fewer splashes

- Longer tundish lifetime due to avoidance of open stream operation

\subsection{Positioning}

Manufacture of the ladle has high tolerances and ladles are moving and rotating. Therefore the position of the shroud cannot be determined as fixed positions. A robot solution has to identify the position where the shroud should be attached.

We are using a target plate with 4 different circles. With these circles, we can easily identify the general orientation of the plate and with 4 circles, we have 1 redundant circle to get the complete $3 d$-position of the target plate (Figure 3).

To ensure a very robust system that is not affected by dirt so easily, the circles are milled into the plate and have a width of $3 \mathrm{~mm}$.

To be able to detect the position of an object in space, two stereo cameras are necessary (like a human being needs two eyes).

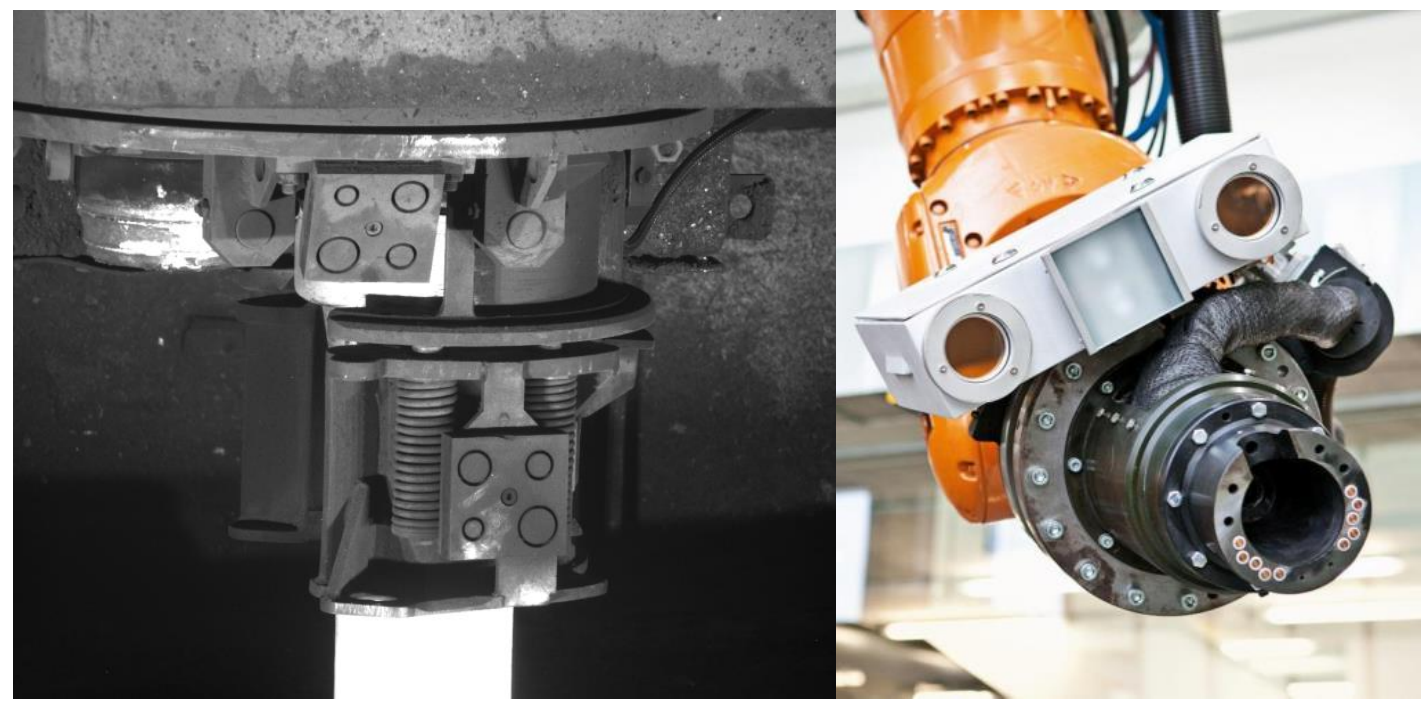

Figure 3. Target plate and stereo cameras for positioning system

For the operation at customer, we use

- One target plate per ladle to get actual ladle position

- One target plate per holder to get actual position of the holder. The position of the holder and the ladle general position may change due to thermic effects.

- We use two target plates per SOS holder, one to detect the rotation of the rotary slide gate and the second plate for detection of oxygen connection.

- Additionally to the stereo cameras on LiquiRob, we use fixed cameras on the ladle operation platform to have redundant target plate recognition and to save time in finding the target position. 


\section{PRE-TESTS}

To guarantee a very short installation time and proper working of the new system from beginning, we performed some pre-tests at the plant.

\subsection{Temperature test}

For the design of the shroud holder, we needed to know the actual temperature condition on ladle. We measured the temperature of the shroud and shroud holder in different casting conditions with thermographic cameras and used these temperatures as input values for our temperature simulations of the shroud holder (Figure 4).
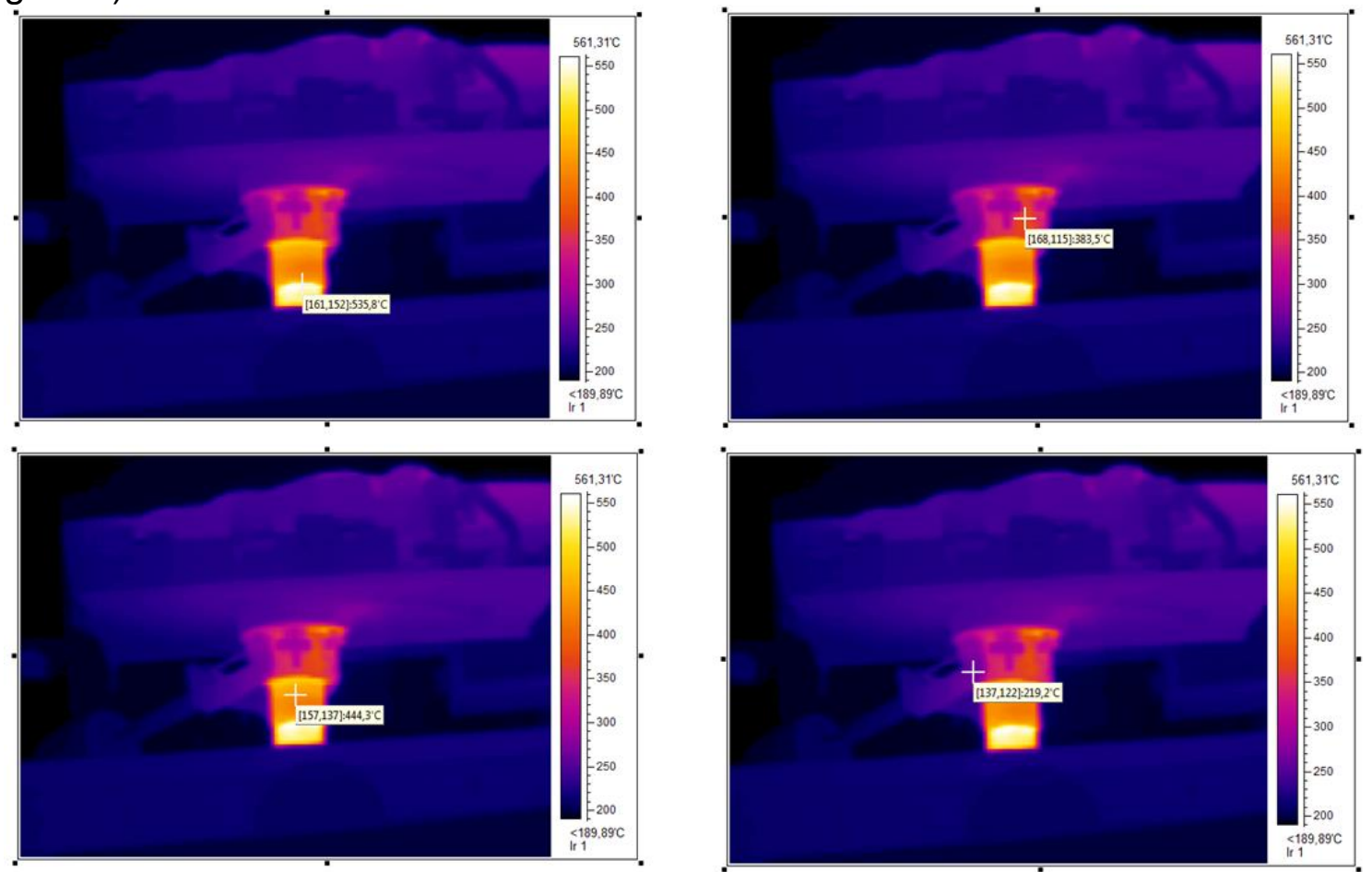

Figure 4. Temperature measurements of shrouds during operation

\subsection{Bayonet test}

Our simulation showed good results for the temperature behavior of the shroud holder. Nevertheless we wanted to test the system under operating conditions prior to first automatic use with LiquiRob.

For the first test, a shroud was mounted manually with a prototype bayonet on the ladle tilting stand. We used a shorter shroud to be able to do the ladle journey with shroud attached. The first time, the complete ladle was cast with a new shroud holder.

These tests have proven (Figure 5)that the shroud holder is not affected by any vibration nor by the temperature condition over the tundish. During standard LiquiRob operation, in one case where there was a malfunction of slide gate, even the pouredin shroud holder held the shroud tight. 


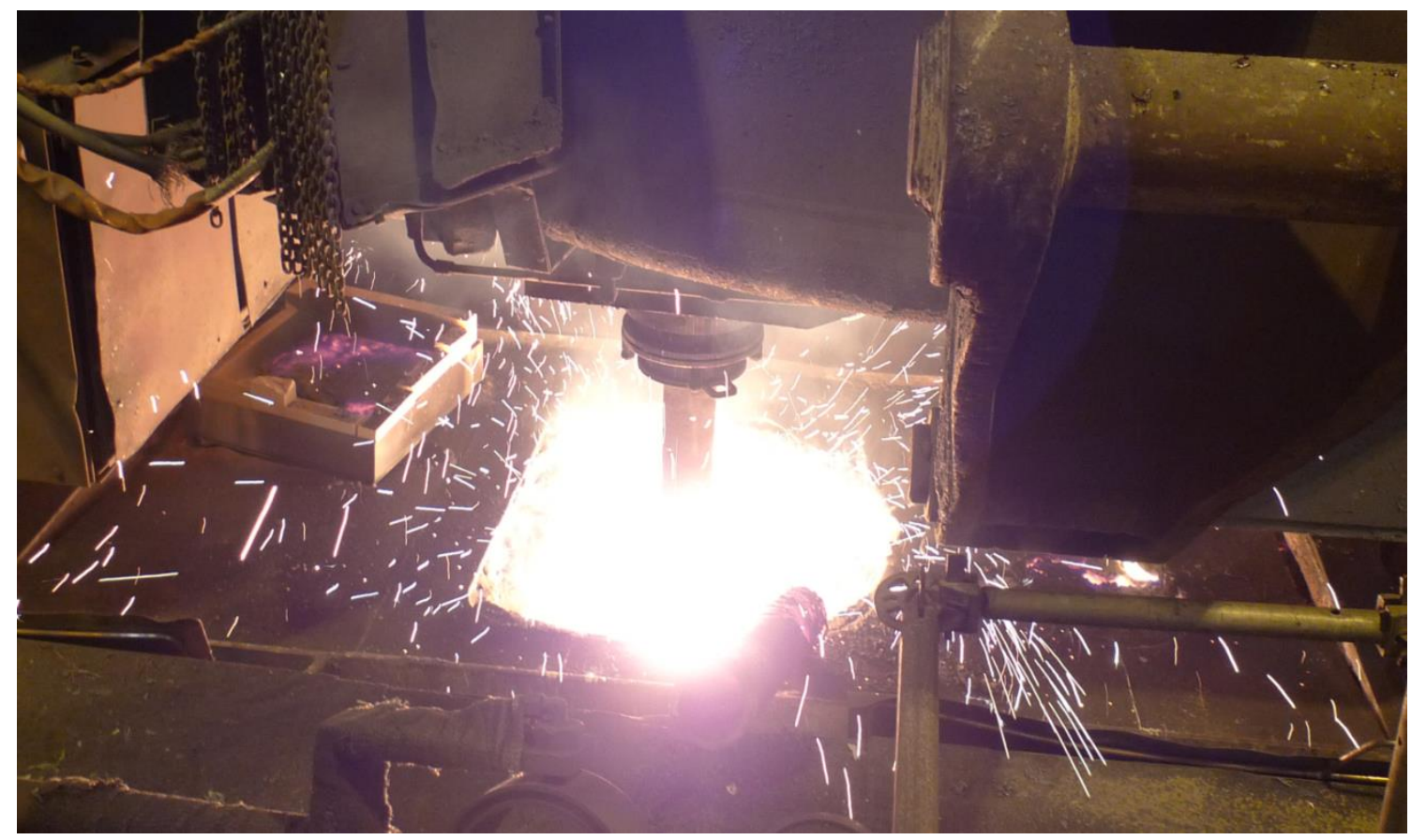

Figure 5. Test of prototype shroud holder under casting conditions.

\section{ENGINEERING AND SIMULATION}

\subsection{Software simulation}

In general all our LiquiRob projects are completely simulated in 3d (Figure 6) to find the optimum position for the robot, to prove the accessibility of all tools, handle tasks and check for collisions.

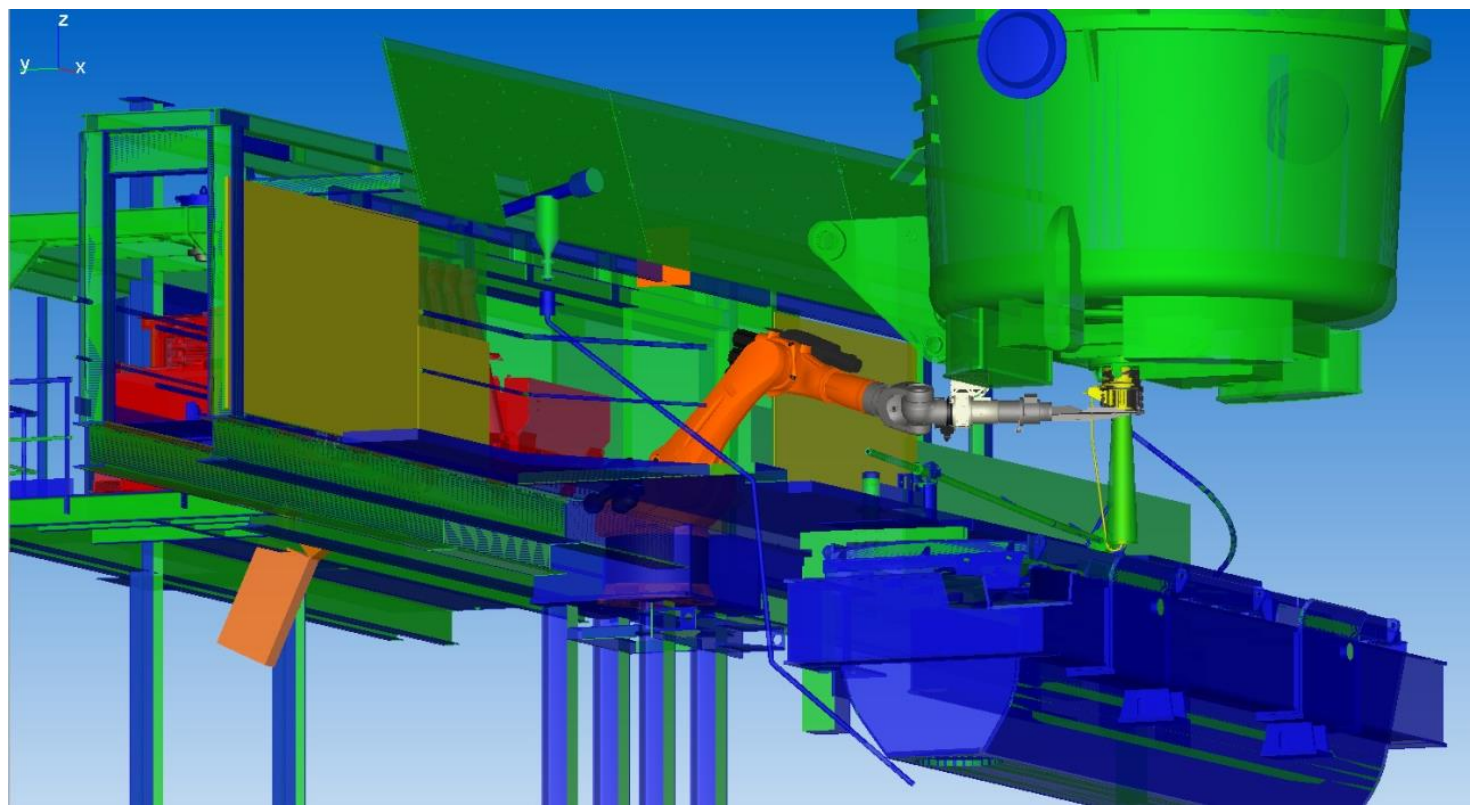

Figure 6. 3d-Simulation 


\subsection{Model in Mechatronic Lab}

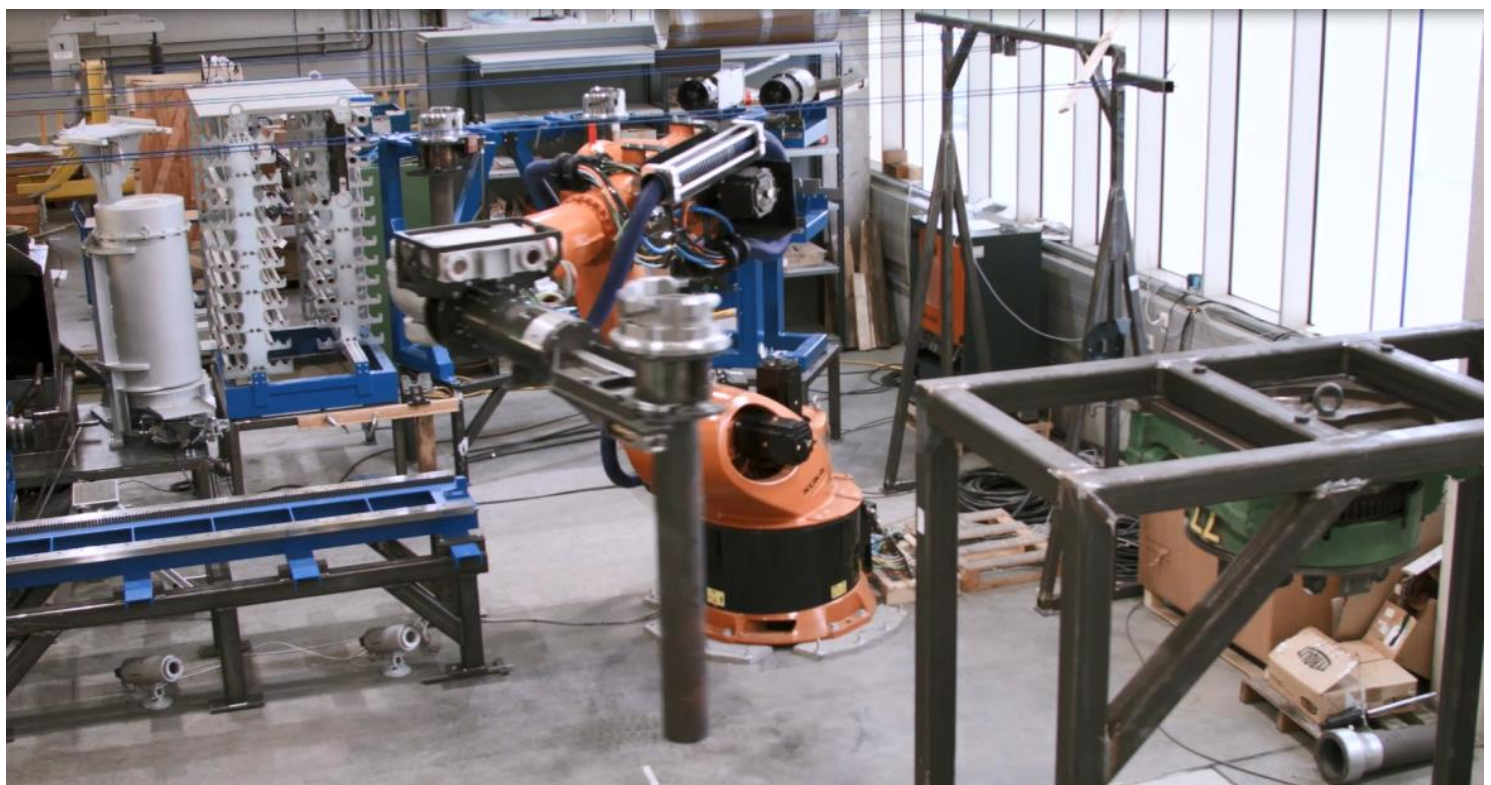

Figure 6. Test assembly in Primetals mechatronic lab

Even with $3 d$ simulation, we assemble the LiquiRob with all tools, targets, magazines and limitations in our maintenance shop. For buyer and seller, we offer these advantages:

- All programming (teach-in) of the robot is done in this laboratory setup.

- Complete automation, like handling of tools, shrouds, fences is set up and tested.

- Complete test of communication of safety PLC with LiquiRob is carried out and reviewed with customer requirements.

- The customer sees LiquiRob working in reality. All functions are tested, the safety concept is verified and the customer can also test the operation of HMI including all failure scenarios - A factory acceptance certificate is usually the result of this test.

- Additionally it offers the chance to pre-train the operators

- All components are tests with real hardware and according software like in the real plant

- Many people are involved in the set-up and testing of a robot. During function tests, not all participants are necessary all the time. If these tests are done in-house, people are called in when they are needed.

\subsection{Safety}

Safety is one of the most important topics in robot application. There are general safety standards that have to be fulfilled like ISO 10218 [1], [3] (Robots for industrial environments - Safety requirements) as well as customer-specific safety features (like specific rules for door opening).

The complete safety concept is handled in safety PLC. This PLC also takes care of the complete communication with other PLCs e.g. for lifting and turning of ladle and slide gate movement.

The realized safety concept comprises:

- 6 doors for protection of the LiquiRob operation cell 
- 3 pneumatic cylinders for tool exchanger, locking for shroud gripper and the door of the shroud heater

- 2 oxygen panels

- Complete LiquiRob control

- Movement of magazine and disposal box

- Control of the drive for the $5 \mathrm{~m}$ sliding door to close robot cell. When an operator is entering the robot cell, this door prevents him from going out to the tundish. Furthermore, it prevents objects from falling on mold level when tundish is not in position.

- Automatic ignition of natural gas in heater

\section{INSTALLATION AND START-UP}

Weeks before shutdown, the first structure works, like reinforcement of ladle operator platform for shroud magazine car and LiquiRob, had been completed.

During the hot phase with quick installation of all systems, up to 25 people were working on 5 areas at the same time ( 3 levels of ladle operation platform, in electric room and in programming room)

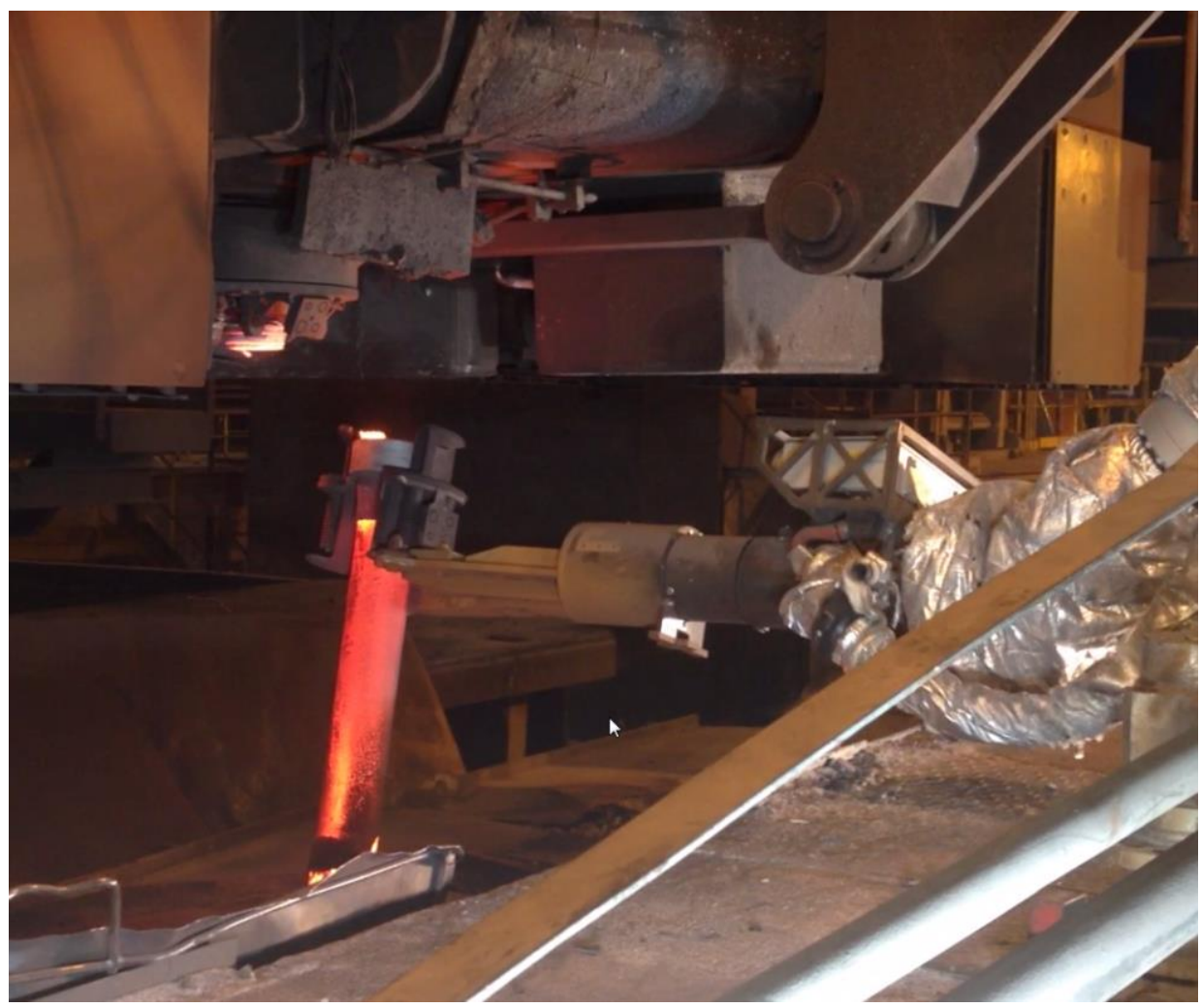

Figure 7. LiquiRob in operation 


\section{Operational Results}

For better training of the operators at the beginning, they had to confirm different steps of LiquiRob movement. As they got more used to the robot, more and more of these holding points were cancelled so that LiquiRob is doing full automatic operation.

A typical performance of the system for a weekly production is:

Casting of 160 ladles

All shrouds have been fully automatically connected by LiquiRob

320 shroud operations without any error

All other tasks have been fulfilled in the meantime

\section{SUMMARY}

With the new bayonet shroud holder, LiquiRob can be used for many tasks on the ladle operation platform like:

- Shroud manipulation

- Ladle oxygen lancing

- Tundish temperature measurement

- Tundish oxygen measurement

- Tundish hydrogen measurement

- Taking tundish steel samples

The advantages of the new system are:

- An emergency turning of the ladle is always possible because the robot is not permanently connected to the ladle

- Avoidance of accidents due to elimination of operators from ladle operation platform.

- LiquiRob can do additional tasks, not only shroud manipulation

- Time for ladle exchange is always the same.

- The force with which the shroud is pressed against the ladle can be adjusted simply (by the spring load) and is always constant.

- There is no turning or movement of the shroud against the slide gate when this is opening or closing

- The shroud holder customers are not limited to a specially manufactured shroud type / manufacturer

- The mechanics that have to be installed on each ladle are very robust, simple and cheap.

\section{REFERENCES}

1 Wimmer, F. et al.: "A Major Step Ahead in Caster Safety with Extensive Use of Robotics in Continuous Casting Technology"; CCR'04; June 14-15, 2004; Linz, Austria Structure of article's reference: Author(s). Title of the article. Title of the journal. Year; volume(number): pages.

2 Penn, J. et al.: “LiquiRob - A new answer for Caster Safety”; CCC'08; May 26-27, 2008; Linz, Austria 
3 Dr. Martin Hirschmanner, et al:"LiquiRob-Improved safety and systematic procedures on the casting floor using advanced robotics";ECCC'11; June 27 - July 1,2011; Duesseldorf, Germany

4 Juergen Meisel et al.: "Experience and Evolution: 10 Years of robotics in continuous casting technology" 8th ECCC June 23-26 2014, Graz 